\title{
New insights into analytical science in China
}

\author{
Hua Cui ${ }^{1} \cdot$ Lihua Zhang $^{2} \cdot$ Qiankun Zhuang $^{3}$ \\ Published online: 29 April 2019 \\ (C) Springer-Verlag GmbH Germany, part of Springer Nature 2019
}

In recent years, with the increased funding input by the Chinese government on scientific research, the number of researchers in analytical science in China has been growing rapidly, and the research level has been elevating constantly. Great progress has been made in the development of new concepts, principles, methodologies, and biomedical applications in analytical science based on nanotechnology, functional nucleic acids, bimolecular recognition, microfluidic techniques, particularly in the fields of single molecule, single cell and single nanoparticle analysis, in vivo bioanalysis, in vivo imaging, bioassays, and biosensing and hyphenated technologies. With this collection that includes 2 critical reviews, 1 communication and 26 research articles, we present the most recent advances in analytical science in China.

Contributions to the collection include fluorescence, chemiluminescence, surface-enhanced Raman mass spectrometry, chromatography, and electroanalysis. In spectrometry and electroanalysis, various functional nanomaterials such as metal nanomaterials, layered nanomaterials, and quantum dots have been used as nanoluminophores, catalysts, and electrodes to fabricate analytical probes and nanointerfaces in bioassays to facilitate improvements in analytical performance. Various enzymes and functional nucleic acids have also been used for signal amplification and further improvement of analytical performance. In separation science, besides the focus on sample preparation, in order to increase the sensitivity of

Published in the topical collection New Insights into Analytical Science in China with guest editors Lihua Zhang, Hua Cui, and Qiankun Zhuang.

Hua Cui

hcui@ustc.edu.cn

1 Department of Chemistry, University of Science and Technology of China, Jinzhai Road 96, Hefei, China

2 Dalian Institute of Chemical Physics, Chinese Academy of Sciences, National Chromatographic R. \& A. Center, 457 Zhongshan Road, Dalian, Liaoning, China

3 Analytical Chemistry Division, National Natural Science Foundation of China, Beijing, China target or overall components, recently, much effort has been made on how to increase the peak capacity of chromatography or electrophoresis, to match the continuously increasing fast scanning speed of mass spectrometry. The improvement on the ionization and fragmentation efficiency of analytes is also of great significance to boost the identification capacity. Moreover, microfluidic techniques have become more mature to meet the requirements of the real world. Furthermore, with the in-depth applications in the analysis of complex samples, such as omic samples, environmental pollutants and additives in food, the establishment of database, and the design of software to mine the huge data generated in the analysis process are also taken into consideration. All the aforementioned points are included in this special issue.

We are very grateful to all authors for their valuable contributions to this topical collection. We would like to thank the reviewers for their constructive and insightful comments and the editorial team of Analytical and Bioanalytical Chemistry for their professional support and cooperation. We are sure that $\mathrm{ABC}$ readers will appreciate this collection, which will benefit and promote the development of analytical science.

Publisher's note Springer Nature remains neutral with regard to jurisdictional claims in published maps and institutional affiliations. 


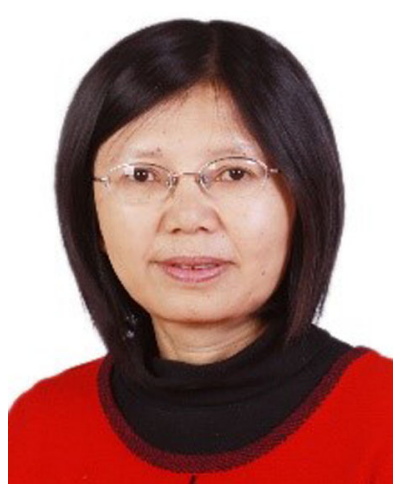

Hua Cui is a full Professor of Analytical Chemistry at the University of Science and Technology of China. She is also an editor of Analytical and Bioanalytical Chemistry and an editorial board member of Analytical Chemistry. Her current research interests are nanochemiluminescence and nanoelectrochemiluminescence and their applications in interdisciplinary fields such as public health, food safety, and environmental monitoring.

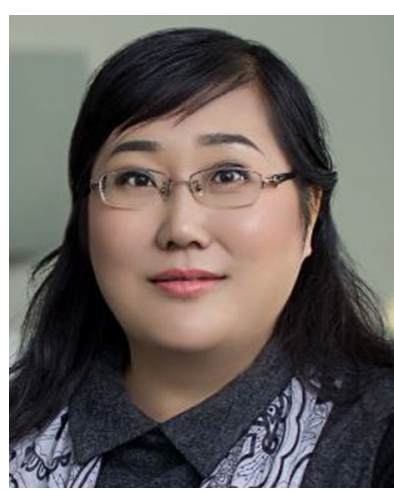

Lihua Zhang is a full Professor of Analytical Chemistry in Dalian Institute of Chemical Physics, Chinese Academy of Sciences. Her current research interests are on the development of LC-MS/ MS-based new methods for proteome qualitation and quantitation and interaction, as well as applications in various fields, such as cell biology, precise medicine, environmental toxicology, and clean energy.

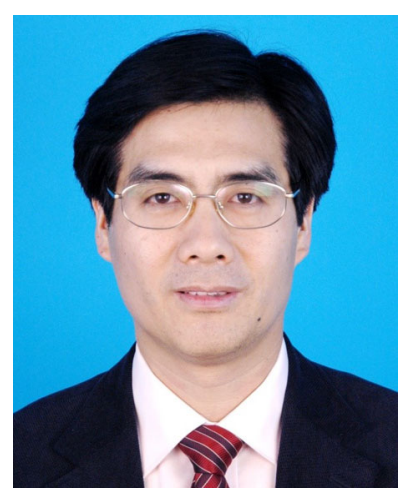

Qiankun Zhuang was previously a Full Professor of Peking University before 2003 and the Director of the Analytical Chemistry Division of National Natural Science Foundation of China (NSFC) from 2003 to 2017. Now, he is Director of the Environmental Chemistry Division of NSFC. 